\title{
A new criterion for assessing llizarov treatment outcomes in nonunion of the tibia
}

\author{
Łukasz Szelerski ${ }^{1}$. Andżelika Pajchert Kozłowska ${ }^{2} \cdot$ Sławomir Żarek ${ }^{1} \cdot$ Radosław Górski $^{1} \cdot$ Karol Mochocki $^{1}$. \\ Maciej Dejnek ${ }^{3} \cdot$ Wiktor Urbański $^{2} \cdot$ Paweł Reichert $^{3} \cdot$ Piotr Morasiewicz $^{2}$ (1)
}

Received: 5 December 2019 / Accepted: 2 August 2020 / Published online: 10 August 2020

(c) The Author(s) 2020

\begin{abstract}
Introduction The purpose of this study was to assess a population of patients with nonunion of the tibia treated with the Ilizarov method in terms of achieved union rates and maintained union rates, determination of re-fracture factors, with a subsequent comparison of our findings with those reported in the available literature.

Materials and methods This study was a retrospective assessment of 102 patients with nonunion of the tibia treated with the Ilizarov method in the period 2008-2015. The assessed parameters were bone union achieved during treatment, duration of stabilization with an Ilizarov external fixator, and maintained bone union at the last follow-up visit.

Results The mean age at the start of treatment was 46.7 years (11-84 years). The mean follow-up period was 7 years (2-12 years). Bone union was achieved in all patients. The mean duration of Ilizarov stabilization in the study group was 7.9 months (2.8-20.7 months). The rate of union maintained at the last follow-up visit was $95.1 \%$.

Conclusions All patients in our study achieved bone union, which constitutes a better outcome than those reported on average in the literature (73.7-100\%). The mean length of time which the Ilizarov external fixator was in place in our patients was 8.3 months, which is consistent with the data from literature. Infection, atrophic nonunion, nonunion in $1 / 3$ distal of tibia, and close surgery technique are risk factors of re-fracture. None of the analyzed studies assessed the proportion of patients with maintained bone union. In our study, maintained bone union was observed in $95.1 \%$ of patients at the follow-up visit at least 2 years after treatment, which indicates excellent long-term treatment outcomes in nonunion of the tibia treated with the Ilizarov method.
\end{abstract}

Keywords Maintained union $\cdot$ Union rates $\cdot$ Nonunion $\cdot$ Tibia $\cdot$ Ilizarov method

\section{Introduction}

Due to the anatomical structure and relatively poor perfusion in the distal third of the leg, fractures in this part of the body-in comparison with other locations-relatively

Piotr Morasiewicz

morasp@poczta.onet.pl

1 Department of Orthopedics and Musculoskeletal Traumatology, Medical University of Warsaw, Lindeya 4, 02-005 Warsaw, Poland

2 Department and Clinic of Orthopaedic and Traumatologic Surgery, Wrocław Medical University, ul. Borowska 213, 50-556 Wrocław, Poland

3 Division of Sport Medicine, Department of Physiotherapy, Faculty of Health Sciences, Wroclaw Medical University, Bartla 5, 51-618 Wrocław, Poland commonly result in disturbed healing and nonunion [1-6]. In tibial fractures, nonunion rates range from 2.5 to $11 \%$ of cases [5, 6]. Ilizarov external fixators are an established technique for treating bone-healing disturbances [1-27]. The treatment aims to achieve bone union and painless, efficient gait, while focusing not only on the immediate outcomes. In fact, one equally important indicator of successful treatment is maintained bone union.

The literature on the subject comprises a number of papers on treating nonunion of the tibia with an Ilizarov external fixator [1-27]. The investigators focused on presenting various surgical techniques $[2,3,5-11,13$, 16-19, 23, 26] and assessing the supportive role of various medications, means, and techniques in achieving bone union $[1,4]$. These studies were predominantly concerned with assessing short-term treatment results [1-27]. For instance, the available literature on assessing Ilizarov 
method treatment outcomes in patients with nonunion of the tibia used such parameters as the rate of union, which is a short-term outcome [1-27]. Some patients who achieve union later develop re-fracture at the pseudarthrosis union site [1, 19], with the re-fracture rates as high as $31.6 \%$ [19]. These patients require retreatment, which translates to poor long-term outcomes.

Some authors who reported re-fracture rates considered them only as a complication, with no significant impact on treatment outcomes [1, 19, 25]. There are no studies on the long-term treatment outcomes (in terms of maintaining bone union over many years of follow-up) in nonunion of the tibia treated with the Ilizarov method. Thus, we would like to introduce a new criterion in assessing long-term outcomes, namely, maintained bone union. We believe the outcomes of treating nonunion of the tibia with the Ilizarov method which should be assessed comprehensively, both in terms of short-term (union rates-achieving union after treatment) and long-term parameters (maintained union rates).

The purpose of this study was to assess a population of patients with nonunion of the tibia treated with the Ilizarov method in terms of achieved union rates and maintained union rates, determination of re-fracture factors, with a subsequent comparison of our findings with those reported in the available literature.

\section{Materials and methods}

This study was a retrospective assessment of 102 patients treated by two of the authors for posttraumatic nonunion of the tibia in the period 2008-2015 (Figs. 1, 2).

The inclusion criteria were nonunion of the tibia treated with an Ilizarov external fixator, at least 2 years after treatment end, investigator access to all medical records, and radiological images relating to the patients' treatment.

102 patients ( 26 women and 76 men) met all the inclusion criteria. Nonunion were caused failed previous internal plate fixation in 70 cases and failed previous intramedullary nail fixation in 32 cases (Table 1). The study was approved by the Local Institutional Review Board. It was single-center study. We analyzed medical and radiological documentation from hospital records.

The surgical procedures were conducted by two experienced orthopedic surgeons. In the case of nonunion located in the proximal two-thirds of the tibial shaft, the Ilizarov

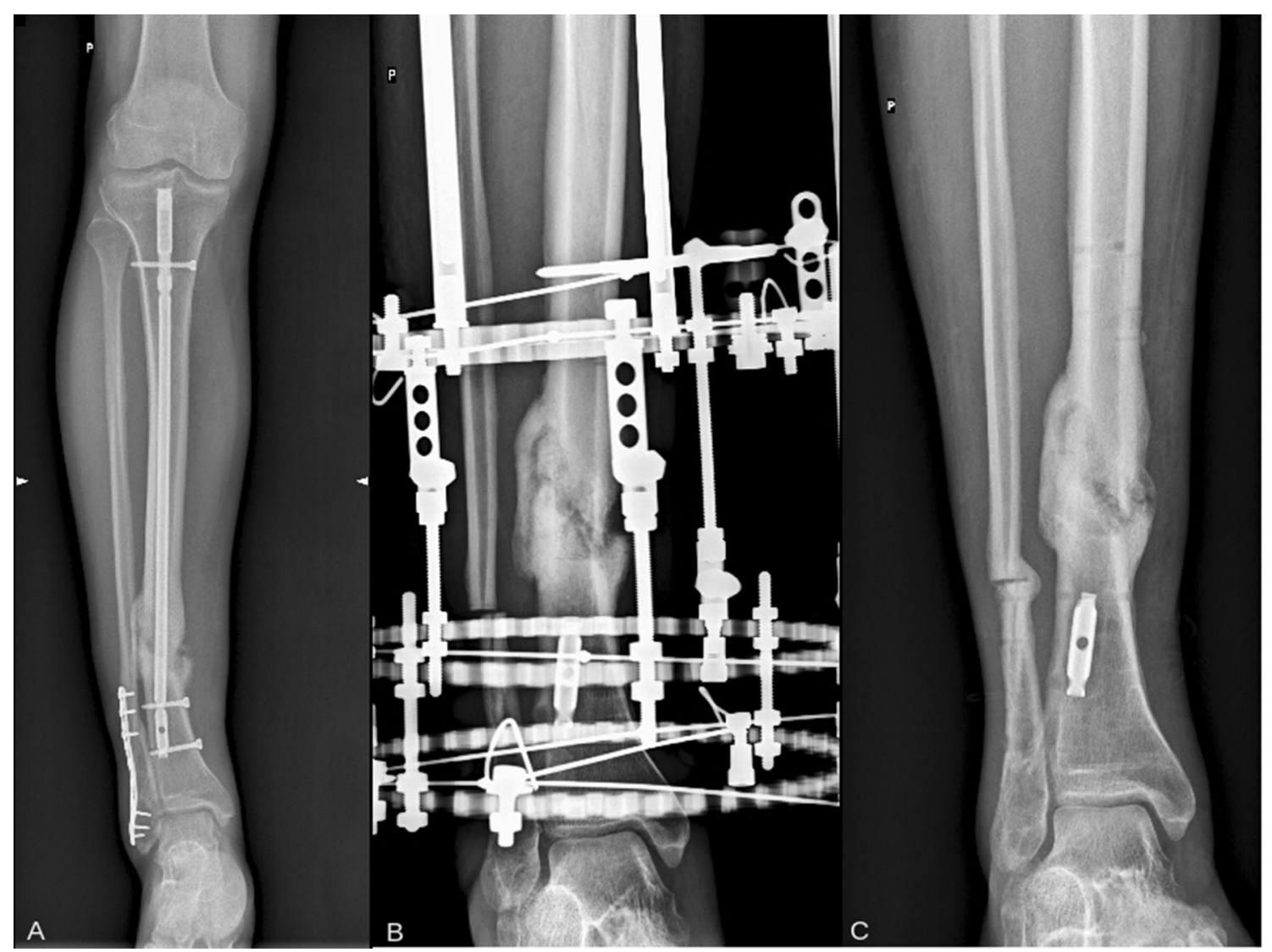

Fig. 1 Patient with tibia nonunion. a X-ray from the pre-treatment period, $\mathbf{b}$ X-ray with Ilizarov apparatus after union, and $\mathbf{c}$ X-ray from the last follow-up visit after 6 years form apparatus removal, confirming maintained union 

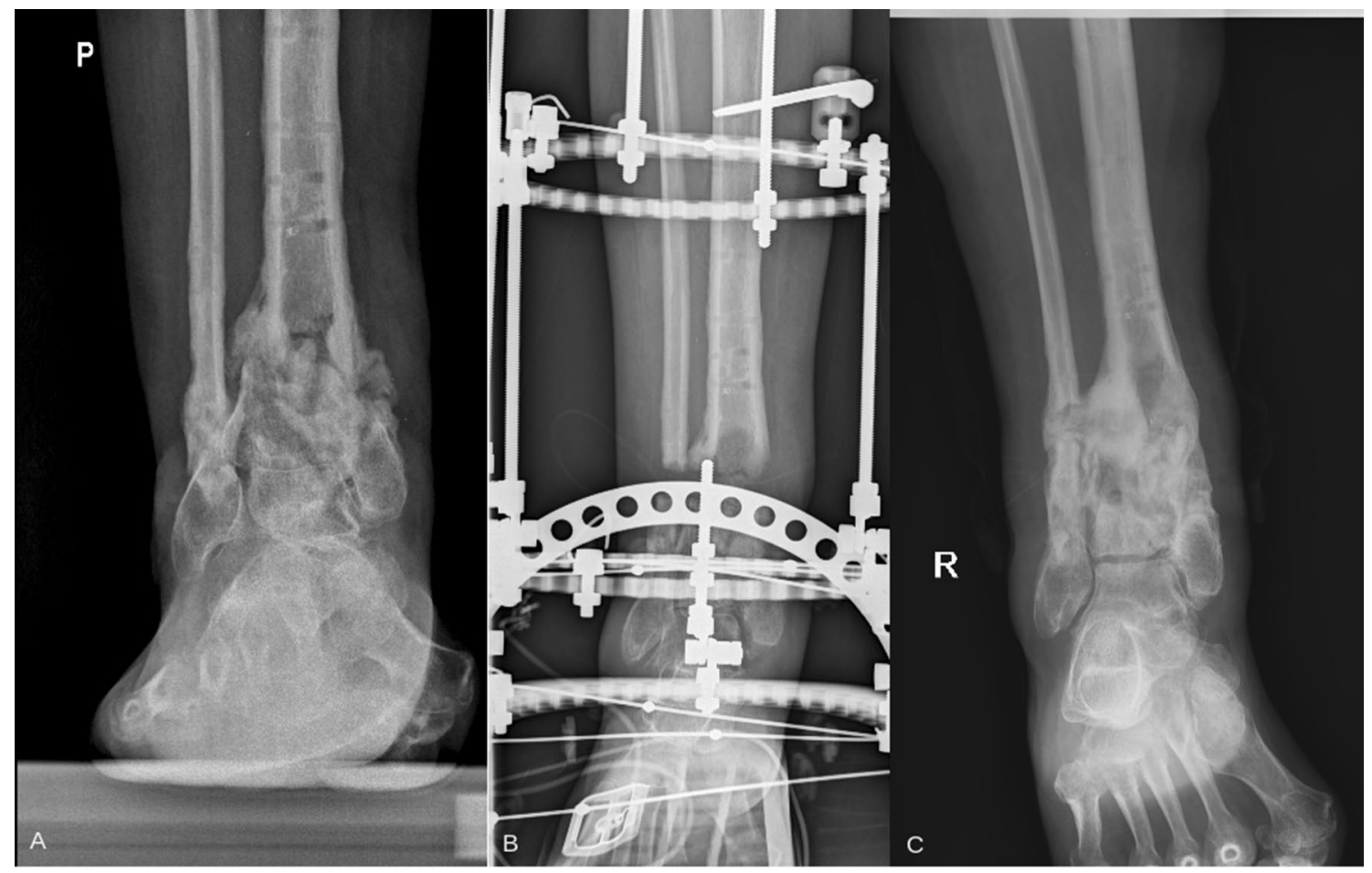

Fig. 2 Patient with tibia nonunion. a X-ray from the pre-treatment period, $\mathbf{b}$ X-ray with Ilizarov apparatus after union, and $\mathbf{c}$ X-ray from the last follow-up visit after 7 years form apparatus removal, confirming maintained union

apparatus consisted of four rings fixed to the tibia and fibula with Kirschner wires. In the case of nonunion of the distal third of the tibia, the Ilizarov apparatus consisted of three rings fixed to the tibia and fibula with Kirschner wires and a foot frame stabilized with three olive Kirschner wires. Tibial nonunion were treated with stabilization and compression, without the use of the bone transport technique. In 73 cases, closed stabilization of nonunion was performed. In 29 patients an open, small bone fragment resection was performed, with adaptation of the nonunion edges and stabilization with the Ilizarov apparatus. We did not use bone grafts. The distal surface of the proximal tibial fragment and the proximal surface of the distal tibial fragment were drilled with Kirschner wires according to Becks's method.

Patient verticalization and gait training with partial weight-bearing on the operated limb and the use of two forearm crutches was initiated on postoperative day one. Clinical and radiographic follow-up visits were conducted in an outpatient setting in 2-6-week intervals. Over the course of treatment, loading of the operated limb was progressive increased until, eventually, the crutches could be discarded as full weight-bearing was achieved.

The Ilizarov external fixator was removed once union of the nonunion was confirmed radiographically and clinically. The radiographic criterion of union was the presence of at least three out of four cortices or trabecular bridging in anteroposterior and lateral views. The clinical criteria were the absence of pain, absence of pathological mobility, and absence of lower leg deformity on dynamization of the Ilizarov apparatus or on forcible attempts at movement at the site of nonunion. Once their Ilizarov external fixator was removed, the patients were advised to walk with two forearm crutches and bear partial weight on the operated limb for 4 weeks. Loading of the limb was gradually increased, depending on the degree of bone remodeling at the site of nonunion visualized with radiography.

Study assessments were based on radiographic images obtained during treatment and at a follow-up visit minimum 2 years after the removal of Ilizarov external fixator. The assessed parameters were bone union achieved during treatment, duration of stabilization with an Ilizarov external fixator, and maintained bone union at the last follow-up visit. The Association for the Study and Application of the Method of Ilizarov (ASAMI) bone score and ASAMI functional score were evaluated as well at the last follow-up visit $[28,29]$.

The statistical analysis was conducted with STATISTICA 13.3 software. The Shapiro-Wilk test was used to evaluate the normality of distribution of all quantitative parameters. The Mann-Whitney $U$ test or Kruskal-Wallis test (ANOVA) was used to calculate differences between groups. The Wilcoxon signed-rank test for paired samples was used for 
Table 1 Patient characteristics

\begin{tabular}{|c|c|c|c|c|c|c|}
\hline Patient number & Sex & Age & Type of nonunion & Location of nonunion & Causes of nonunion & $\begin{array}{l}\text { Surgery } \\
\text { tech- } \\
\text { nique }\end{array}$ \\
\hline 1 & M & 21 & Hypertrophic & 1/3 Mid & Failed previous plate fixation & Close \\
\hline 2 & M & 17 & Hypertrophic & 1/3 Mid & Failed previous intramedullary nail fixation & Close \\
\hline 3 & M & 48 & Hypertrophic & 1/3 Distal & Failed previous plate fixation & Close \\
\hline 4 & M & 41 & Hypertrophic & 1/3 Distal & Failed previous plate fixation & Close \\
\hline 5 & M & 73 & Hypertrophic & 1/3 Distal & Failed previous plate fixation & Open \\
\hline 6 & M & 29 & Hypertrophic & 1/3 Proximal & Failed previous plate fixation & Close \\
\hline 7 & M & 22 & Hypertrophic & 1/3 Distal & Failed previous plate fixation & Close \\
\hline 8 & M & 62 & Atrophic & 1/3 Distal & Failed previous plate fixation & Open \\
\hline 9 & M & 71 & Hypertrophic & 1/3 Mid & Failed previous intramedullary nail fixation & Open \\
\hline 10 & M & 38 & Hypertrophic & 1/3 Mid & Failed previous intramedullary nail fixation & Close \\
\hline 11 & $\mathrm{~F}$ & 37 & Hypertrophic & 1/3 Distal & Failed previous plate fixation & Open \\
\hline 12 & $\mathrm{~F}$ & 30 & Hypertrophic & 1/3 Mid & Failed previous plate fixation & Open \\
\hline 13 & M & 52 & Hypertrophic & 1/3 Mid & Failed previous intramedullary nail fixation & Open \\
\hline 14 & M & 53 & Hypertrophic & 1/3 Distal & Failed previous plate fixation & Close \\
\hline 15 & M & 30 & Hypertrophic & 1/3 Distal & Failed previous plate fixation & Close \\
\hline 16 & M & 42 & Hypertrophic & 1/3 Distal & Failed previous plate fixation & Close \\
\hline 17 & M & 60 & Hypertrophic & 1/3 Distal & Failed previous plate fixation & Close \\
\hline 18 & M & 54 & Hypertrophic & 1/3 Mid & Failed previous intramedullary nail fixation & Close \\
\hline 19 & M & 66 & Hypertrophic & 1/3 Proximal & Failed previous plate fixation & Close \\
\hline 20 & M & 50 & Hypertrophic & 1/3 Mid & Failed previous intramedullary nail fixation & Close \\
\hline 21 & $\mathrm{~F}$ & 55 & Hypertrophic & 1/3 Distal & Failed previous plate fixation & Close \\
\hline 22 & $\mathrm{~F}$ & 30 & Hypertrophic & 1/3 Proximal & Failed previous plate fixation & Close \\
\hline 23 & M & 51 & Atrophic & 1/3 Distal & Failed previous plate fixation & Close \\
\hline 24 & M & 50 & Hypertrophic & 1/3 Mid & Failed previous intramedullary nail fixation & Open \\
\hline 25 & M & 17 & Hypertrophic & 1/3 Distal & Failed previous plate fixation & Close \\
\hline 26 & M & 11 & Hypertrophic & 1/3 Mid & Failed previous plate fixation & Close \\
\hline 27 & M & 73 & Hypertrophic & 1/3 Mid & Failed previous intramedullary nail fixation & Close \\
\hline 28 & $\mathrm{~F}$ & 71 & Hypertrophic & 1/3 Mid & Failed previous plate fixation & Close \\
\hline 29 & $\mathrm{~F}$ & 23 & Hypertrophic & 1/3 Distal & Failed previous intramedullary nail fixation & Close \\
\hline 30 & $\mathrm{~F}$ & 55 & Atrophic & 1/3 Proximal & Failed previous plate fixation & Open \\
\hline 31 & $\mathrm{~F}$ & 60 & Hypertrophic & 1/3 Mid & Failed previous intramedullary nail fixation & Close \\
\hline 32 & M & 33 & Hypertrophic & 1/3 Distal & Failed previous plate fixation & Open \\
\hline 33 & M & 47 & Hypertrophic & 1/3 Mid & Failed previous intramedullary nail fixation & Open \\
\hline 34 & M & 33 & Atrophic & 1/3 Distal & Failed previous plate fixation & Close \\
\hline 35 & $\mathrm{~F}$ & 23 & Hypertrophic & 1/3 Mid & Failed previous plate fixation & Close \\
\hline 36 & M & 61 & Hypertrophic & 1/3 Mid & Failed previous intramedullary nail fixation & Close \\
\hline 37 & $\mathrm{~F}$ & 15 & Hypertrophic & 1/3 Distal & Failed previous plate fixation & Close \\
\hline 38 & $\mathrm{~F}$ & 56 & Hypertrophic & 1/3 Distal & Failed previous plate fixation & Close \\
\hline 39 & M & 40 & Atrophic & 1/3 Distal & Failed previous plate fixation & Close \\
\hline 40 & M & 42 & Atrophic & 1/3 Distal & Failed previous plate fixation & Close \\
\hline 41 & M & 60 & Hypertrophic & 1/3 Mid & Failed previous intramedullary nail fixation & Open \\
\hline 42 & M & 34 & Atrophic & 1/3 Proximal & Failed previous plate fixation & Close \\
\hline 43 & $\mathrm{~F}$ & 21 & Atrophic & 1/3 Mid & Failed previous intramedullary nail fixation & Open \\
\hline 44 & M & 48 & Hypertrophic & 1/3 Mid & Failed previous plate fixation & Close \\
\hline 45 & M & 41 & Hypertrophic & 1/3 Mid & Failed previous intramedullary nail fixation & Close \\
\hline 46 & M & 73 & Hypertrophic & 1/3 Distal & Failed previous plate fixation & Close \\
\hline 47 & M & 29 & Hypertrophic & 1/3 Distal & Failed previous plate fixation & Close \\
\hline 48 & M & 22 & Hypertrophic & 1/3 Distal & Failed previous plate fixation & Close \\
\hline
\end{tabular}


Table 1 (continued)

\begin{tabular}{|c|c|c|c|c|c|c|}
\hline Patient number & Sex & Age & Type of nonunion & Location of nonunion & Causes of nonunion & $\begin{array}{l}\text { Surgery } \\
\text { tech- } \\
\text { nique }\end{array}$ \\
\hline 49 & $\mathrm{~F}$ & 62 & Atrophic & 1/3 Proximal & Failed previous plate fixation & Open \\
\hline 50 & $\mathrm{~F}$ & 71 & Hypertrophic & 1/3 Distal & Failed previous plate fixation & Close \\
\hline 51 & M & 77 & Hypertrophic & 1/3 Distal & Failed previous plate fixation & Close \\
\hline 52 & M & 38 & Hypertrophic & 1/3 Mid & Failed previous intramedullary nail fixation & Open \\
\hline 53 & M & 37 & Hypertrophic & 1/3 Mid & Failed previous intramedullary nail fixation & Open \\
\hline 54 & M & 30 & Hypertrophic & 1/3 Distal & Failed previous plate fixation & Close \\
\hline 55 & $\mathrm{~F}$ & 52 & Hypertrophic & 1/3 Mid & Failed previous intramedullary nail fixation & Open \\
\hline 56 & $\mathrm{~F}$ & 53 & Hypertrophic & 1/3 Mid & Failed previous intramedullary nail fixation & Close \\
\hline 57 & M & 30 & Hypertrophic & 1/3 Distal & Failed previous plate fixation & Close \\
\hline 58 & M & 42 & Hypertrophic & 1/3 Distal & Failed previous plate fixation & Close \\
\hline 59 & M & 56 & Hypertrophic & 1/3 Distal & Failed previous plate fixation & Close \\
\hline 60 & M & 54 & Hypertrophic & 1/3 Distal & Failed previous intramedullary nail fixation & Close \\
\hline 61 & M & 66 & Hypertrophic & 1/3 Mid & Failed previous plate fixation & Close \\
\hline 62 & M & 50 & Hypertrophic & 1/3 Proximal & Failed previous plate fixation & Close \\
\hline 63 & M & 55 & Hypertrophic & 1/3 Mid & Failed previous intramedullary nail fixation & Close \\
\hline 64 & $\mathrm{~F}$ & 30 & Atrophic & 1/3 Distal & Failed previous plate fixation & Open \\
\hline 65 & $\mathrm{~F}$ & 60 & Hypertrophic & 1/3 Proximal & Failed previous plate fixation & Close \\
\hline 66 & M & 54 & Atrophic & 1/3 Distal & Failed previous plate fixation & Close \\
\hline 67 & M & 51 & Atrophic & 1/3 Mid & Failed previous intramedullary nail fixation & Close \\
\hline 68 & $\mathrm{~F}$ & 59 & Hypertrophic & 1/3 Distal & Failed previous plate fixation & Close \\
\hline 69 & M & 64 & Hypertrophic & 1/3 Mid & Failed previous intramedullary nail fixation & Close \\
\hline 70 & M & 54 & Hypertrophic & 1/3 Mid & Failed previous intramedullary nail fixation & Close \\
\hline 71 & $\mathrm{~F}$ & 45 & Hypertrophic & 1/3 Mid & Failed previous plate fixation & Close \\
\hline 72 & M & 61 & Hypertrophic & 1/3 Distal & Failed previous plate fixation & Open \\
\hline 73 & $\mathrm{~F}$ & 34 & Atrophic & 1/3 Proximal & Failed previous plate fixation & Close \\
\hline 74 & M & 48 & Atrophic & 1/3 Mid & Failed previous intramedullary nail fixation & Open \\
\hline 75 & M & 29 & Hypertrophic & 1/3 Distal & Failed previous plate fixation & Open \\
\hline 76 & $\mathrm{~F}$ & 51 & Hypertrophic & 1/3 Proximal & Failed previous plate fixation & Close \\
\hline 77 & M & 53 & Atrophic & 1/3 Distal & Failed previous plate fixation & Close \\
\hline 78 & M & 15 & Hypertrophic & 1/3 Mid & Failed previous intramedullary nail fixation & Close \\
\hline 79 & M & 76 & Hypertrophic & 1/3 Mid & Failed previous intramedullary nail fixation & Close \\
\hline 80 & M & 64 & Hypertrophic & 1/3 Distal & Failed previous plate fixation & Close \\
\hline 81 & M & 53 & Hypertrophic & 1/3 Distal & Failed previous plate fixation & Close \\
\hline 82 & M & 77 & Hypertrophic & 1/3 Distal & Failed previous plate fixation & Close \\
\hline 83 & M & 25 & Hypertrophic & 1/3 Mid & Failed previous intramedullary nail fixation & Open \\
\hline 84 & $\mathrm{~F}$ & 25 & Atrophic & 1/3 Distal & Failed previous plate fixation & Close \\
\hline 85 & $\mathrm{~F}$ & 62 & Hypertrophic & 1/3 Proximal & Failed previous plate fixation & Open \\
\hline 86 & M & 54 & Atrophic & 1/3 Distal & Failed previous plate fixation & Close \\
\hline 87 & M & 48 & Hypertrophic & 1/3 Distal & Failed previous plate fixation & Close \\
\hline 88 & M & 45 & Hypertrophic & 1/3 Distal & Failed previous plate fixation & Close \\
\hline 89 & M & 53 & Hypertrophic & 1/3 Mid & Failed previous intramedullary nail fixation & Open \\
\hline 90 & M & 51 & Hypertrophic & 1/3 Distal & Failed previous plate fixation & Close \\
\hline 91 & $\mathrm{~F}$ & 74 & Hypertrophic & 1/3 Distal & Failed previous plate fixation & Close \\
\hline 92 & M & 27 & Atrophic & $1 / 3 \mathrm{Mid}$ & Failed previous intramedullary nail fixation & Open \\
\hline 93 & M & 77 & Hypertrophic & 1/3 Proximal & Failed previous plate fixation & Open \\
\hline 94 & M & 36 & Hypertrophic & 1/3 Distal & Failed previous plate fixation & Open \\
\hline 95 & M & 36 & Hypertrophic & 1/3 Mid & Failed previous intramedullary nail fixation & Open \\
\hline 96 & M & 29 & Hypertrophic & 1/3 Distal & Failed previous plate fixation & Close \\
\hline
\end{tabular}


Table 1 (continued)

\begin{tabular}{|c|c|c|c|c|c|c|c|c|}
\hline Patient number & Sex & Age & Type of nonunion & Location of nonunion & Causes & nonunion & & $\begin{array}{l}\text { Surgery } \\
\text { tech- } \\
\text { nique }\end{array}$ \\
\hline 97 & M & 34 & Atrophic & 1/3 Distal & Failed & ious plate fixation & & Close \\
\hline 98 & M & 38 & Hypertrophic & 1/3 Distal & Failed & ious plate fixation & & Close \\
\hline 99 & M & 48 & Hypertrophic & 1/3 Distal & Failed & ious plate fixation & & Open \\
\hline 100 & M & 38 & Hypertrophic & 1/3 Distal & Failed $_{1}$ & ious plate fixation & & Close \\
\hline 101 & $\mathrm{~F}$ & 65 & Atrophic & 1/3 Distal & Failed & ious plate fixation & & Close \\
\hline 102 & M & 59 & Hypertrophic & 1/3 Mid & Failed $_{1}$ & ious intramedullary & ation & Open \\
\hline Patient number & & & AMI bone score & ASAMI funct & al score & Refracture & Follo & up (years) \\
\hline 1 & & & ellent & Excellent & & No & 5.6 & \\
\hline 2 & & & ellent & Excellent & & No & 11.7 & \\
\hline 3 & & & ellent & Excellent & & No & 11.3 & \\
\hline 4 & & & & Good & & No & 11.2 & \\
\hline 5 & & & ellent & Excellent & & No & 11 & \\
\hline 6 & & & ellent & Good & & No & 10.9 & \\
\hline 7 & & & ellent & Excellent & & No & 10.5 & \\
\hline 8 & & & ellent & Good & & No & 10.4 & \\
\hline 9 & & & ellent & Excellent & & No & 10.1 & \\
\hline 10 & & & ellent & Excellent & & No & 10 & \\
\hline 11 & & & ellent & Good & & No & 9.6 & \\
\hline 12 & & & ellent & Good & & No & 9.7 & \\
\hline 13 & & & ellent & Excellent & & No & 9.4 & \\
\hline 14 & & & ellent & Excellent & & No & 9.6 & \\
\hline 15 & & & ellent & Excellent & & No & 9.5 & \\
\hline 16 & & & ellent & Excellent & & No & 9.2 & \\
\hline 17 & & & ellent & Good & & No & 9 & \\
\hline 18 & & & ellent & Good & & No & 8.2 & \\
\hline 19 & & & ellent & Excellent & & No & 7.7 & \\
\hline 20 & & & & Good & & No & 8.5 & \\
\hline 21 & & & ellent & Excellent & & No & 8.4 & \\
\hline 22 & & & ellent & Excellent & & No & 10.9 & \\
\hline 23 & & & ellent & Excellent & & No & 8.5 & \\
\hline 24 & & & ellent & Good & & No & 11.7 & \\
\hline 25 & & & ellent & Excellent & & No & 11.2 & \\
\hline 26 & & & ellent & Excellent & & No & 11.5 & \\
\hline 27 & & & ellent & Excellent & & No & 11.7 & \\
\hline 28 & & & ellent & Excellent & & No & 12 & \\
\hline 29 & & & ellent & Good & & No & 11.7 & \\
\hline 30 & & & ellent & Excellent & & No & 11.5 & \\
\hline 31 & & & ellent & Good & & No & 11.7 & \\
\hline 32 & & & ellent & Excellent & & No & 2.5 & \\
\hline 33 & & & ellent & Excellent & & No & 10.6 & \\
\hline 34 & & & ellent & Good & & No & 11.6 & \\
\hline 35 & & & ellent & Good & & No & 12 & \\
\hline 36 & & & ellent & Excellent & & No & 11.9 & \\
\hline 37 & & & ellent & Excellent & & No & 10.8 & \\
\hline 38 & & & & Good & & No & 11.8 & \\
\hline 39 & & & ellent & Excellent & & No & 10.5 & \\
\hline 40 & & & ellent & Excellent & & No & 11.6 & \\
\hline
\end{tabular}


Table 1 (continued)

\begin{tabular}{|c|c|c|c|c|}
\hline Patient number & ASAMI bone score & ASAMI functional score & Refracture & Follow-up (years) \\
\hline 41 & Excellent & Excellent & No & 11.5 \\
\hline 42 & Good & Good & No & 10.8 \\
\hline 43 & Excellent & Excellent & No & 11.8 \\
\hline 44 & Excellent & Excellent & No & 7.41 \\
\hline 45 & Good & Good & No & 5.3 \\
\hline 46 & Excellent & Excellent & No & 2.01 \\
\hline 47 & Excellent & Good & No & 8.98 \\
\hline 48 & Excellent & Excellent & No & 8.54 \\
\hline 49 & Excellent & Good & No & 10.01 \\
\hline 50 & Excellent & Excellent & No & 7.03 \\
\hline 51 & Excellent & Excellent & No & 5.96 \\
\hline 52 & Excellent & Good & No & 5.87 \\
\hline 53 & Excellent & Good & No & 3.99 \\
\hline 54 & Excellent & Excellent & No & 4.72 \\
\hline 55 & Excellent & Excellent & No & 3.44 \\
\hline 56 & Excellent & Excellent & No & 3.68 \\
\hline 57 & Excellent & Excellent & No & 3.5 \\
\hline 58 & Excellent & Good & No & 2.39 \\
\hline 59 & Excellent & Good & No & 5.86 \\
\hline 60 & Excellent & Excellent & No & 4.28 \\
\hline 61 & Good & Good & No & 3.13 \\
\hline 62 & Excellent & Excellent & No & 2.56 \\
\hline 63 & Excellent & Excellent & No & 8.53 \\
\hline 64 & Excellent & Excellent & No & 2.06 \\
\hline 65 & Excellent & Excellent & No & 2.31 \\
\hline 66 & Good & Good & No & 5.81 \\
\hline 67 & Excellent & Excellent & No & 2.4 \\
\hline 68 & Excellent & Good & No & 2.21 \\
\hline 69 & Poor & Poor & Yes & 2.85 \\
\hline 70 & Excellent & Excellent & No & 2.25 \\
\hline 71 & Excellent & Excellent & No & 2.07 \\
\hline 72 & Excellent & Good & No & 2.51 \\
\hline 73 & Excellent & Excellent & No & 2.16 \\
\hline 74 & Excellent & Excellent & No & 2.3 \\
\hline 75 & Excellent & Excellent & No & 4.4 \\
\hline 76 & Excellent & Excellent & No & 6.33 \\
\hline 77 & Poor & Poor & Yes & 10.5 \\
\hline 78 & Excellent & Excellent & No & 7.5 \\
\hline 79 & Excellent & Excellent & No & 8.5 \\
\hline 80 & Excellent & Excellent & No & 4.5 \\
\hline 81 & Excellent & Good & No & 7.66 \\
\hline 82 & Excellent & Excellent & No & 8 \\
\hline 83 & Excellent & Excellent & No & 6.66 \\
\hline 84 & Poor & Poor & Yes & 6.5 \\
\hline 85 & Excellent & Excellent & No & 6.5 \\
\hline 86 & Excellent & Good & No & 6.58 \\
\hline 87 & Excellent & Excellent & No & 5.5 \\
\hline 88 & Excellent & Excellent & No & 4.5 \\
\hline 89 & Excellent & Excellent & No & 4.58 \\
\hline 90 & Excellent & Excellent & No & 2.58 \\
\hline
\end{tabular}


Table 1 (continued)

\begin{tabular}{lllll}
\hline Patient number & ASAMI bone score & ASAMI functional score & Refracture & Follow-up (years) \\
\hline 91 & Excellent & Good & No & 3.33 \\
92 & Excellent & Excellent & No & 2.5 \\
93 & Good & Good & No & 2.58 \\
94 & Excellent & Excellent & No & 2.5 \\
95 & Excellent & Excellent & No & 2.66 \\
96 & Excellent & Excellent & Yes & 6.42 \\
97 & Poor & Poor & No & 2.75 \\
98 & Excellent & Excellent & No & 2.5 \\
99 & Excellent & Excellent & No & 2.5 \\
100 & Excellent & Excellent & Yes & 2.66 \\
101 & Poor & Poor & No & 2.58 \\
102 & Excellent & Excellent & & 2.58 \\
\hline
\end{tabular}

repeated measurements; potential correlation was assessed with Spearman's rank correlation coefficient (rho). The level of statistical significance was set at $p<0.05$.

\section{Results}

A total of 102 patients were assessed (Table 1). The mean age at the start of treatment was 46.5 years (11-77 years, SD 17.35). The mean follow-up period was 7 years (2-12 years, SD 2.23). Bone union was achieved in all patients. The mean duration of Ilizarov stabilization in the study group was 7.9 months (2.8-20.7 months, SD 4.29). ASAMI bone scores were excellent in 88 cases, good in nine cases, and poor in five case. ASAMI functional scores were excellent in 67 cases, good in 30 cases, and poor in five case. The rate of union maintained at the last follow-up visit was $95.1 \%$ (i.e., bone union was maintained in 97 out of 102 evaluated patients). Five persons developed a re-fracture of the healed site and required restabilization with an Ilizarov external fixator. Four out of five patients with re-fracture had infected, atrophic nonunion in 1/3 distal of tibia. All of patients with re-fracture had close surgery technique. The results of statistical analysis showed that infection $(p=0.032)$, atrophic nonunion $(p=0.021)$, nonunion in $1 / 3$ distal of tibia $(p=0.038)$, and close surgery technique $(p=0.017)$ are the independent risk factors of re-fracture. In the hypertrophic pseudarthrosis group, the median time to union (195.0 days) was significantly shorter than in the atrophic pseudarthrosis group (299.0 days), $p=0.021$. The mean time of re-fracture was 2 months after Ilizarov fixator removal (1-6 months). The mean time to union after Ilizarov re-fixation was 9.4 months (4.8-12.7 months, SD 3.34). All of the re-fracture patients had poor results in ASAMI Bone Score and ASAMI Functional Score.
At least one risk factor for disturbance in bone healing has been reported in 22 patients. The following risk factors were considered: corticosteroid therapy, smoking, alcohol dependence, diabetes mellitus, and advanced lower-extremity vascular disease. There was no significant difference in time to union between the group of patients with risk factor for disturbance in fracture healing and the group without risk factors $(p=0.827)$.

\section{Discussion}

Nonunion of the tibia is a common treatment complication in tibial fractures [3-6]. Despite advancements in surgical techniques, nonunion remains a serious therapeutic issue [2-7, 9, 10, 12-19], as it often requires comprehensive surgical treatment involving resection of damaged bone and soft tissues, excision of the focus of infection, and secondary elongation and realignment of the affected bone segment following bone transport [1-7, 9-19]. While evaluating treatment outcomes in nonunion of the tibia, the majority of authors focus on whether or not bone union was achieved [1-27, 30-33]. However, bone union may be only short term, as some patients develop re-fracture [1, 19], which necessitate further treatment and adversely affect long-term outcomes.

The purpose of our study was to assess the rates of achieved (short term, achieving union after treatment) and maintained (long term) bone union and determination of re-fracture factors in a group of patients treated at the Our Clinic, to review the literature on the treatment of nonunion of the tibia with Ilizarov external fixators, and to compare the results reported in the available literature with our findings. We include a larger cohort of tibial nonunion cases (102), whether aseptic or infected, to study the incidence of re-fracture after long-term follow-up of Ilizarov fixation and to address different risk factors, treatment methods, and the 
effect of re-fracture on final outcome in comparison to cases with maintained bone union.

Yin et al. conducted a meta-analysis of 24 studies in a total of 590 patients treated with an Ilizarov external fixator due to infected femoral or lower leg nonunion [1] and showed a union rate of $97.8 \%$. Six of the analyzed studies considered re-fracture as one of treatment complications and reported it in a mean of $4 \%$ of cases [1]. However, the authors did not include any information on when the re-fracture occurred, its treatment, or its effect on the treatment outcome. The proportion of patients in whom bone union was successfully maintained was likewise not included [1]. Peng et al. presented 58 cases of treating infected nonunion of the tibia with Ilizarov bone transport with an antibiotic-loaded bone cement spacer. Bone union was achieved in all patients [2]. Ilizarov fixator were removed after a 10.6 months. They have no re-fracture in follow-up. McNally et al. evaluated 79 patients with nonunion of the tibia treated with the Ilizarov method implemented with various surgical techniques [19]. Depending on the surgical technique used, primary bone union was achieved in 73.7-96.2\% of patients, with the refracture rate in the monofocal compression group as high as $31.6 \%$ [19]. Further treatment helped to achieve union in $100 \%$ of cases. The authors did not assess the proportion of patients who achieved maintained bone union [19]. Laursen assessed 16 patients with nonunion of the tibia treated with the Ilizarov method and reported bone union in $93.8 \%$ of patients after a mean treatment duration of 6 months, with no recorded cases of re-fracture [25].

In the literature reports about tibial nonunion treatment, only a few authors give short information about protocol in preventing re-fracture $[5,7,12,13,17,21]$. Abuomira et al. evaluated 55 patients treated with circular frames due to nonunion of the tibia [7]. Bone union was achieved in $89 \%$ of cases, with the mean treatment duration of 13 months. They removed external fixator when three or four cortical was seen in radiographs. After external fixator removal, patients walked with partial weight-bearing for 4-6 weeks. The rates of maintained union were not reported [7]. Madhusudhan et al. evaluated 22 patients treated with Ilizarov external fixators due to nonunion of the tibia. Bone union was achieved in $81.8 \%$ of patients [12]. They removed external fixator when union was seen in radiographs. After external fixator removal patients walked with functional cast brace for a few weeks [12]. Magadum, who analyzed treatment outcomes in 25 patients with nonunion of the tibia treated with an Ilizarov external fixator, reported bone union in $96 \%$ of patients [13]. After Ilizarov fixator removal patients walked with cast for a 6 weeks [13]. Meleppuram et al. achieved bone union in $100 \%$ out of 42 patients with nonunion of the tibia treated with an Ilizarov external fixator [5]. They removed fixator when the nonunion was corticolized on 3 of 4 sides. They used casts for a 2 months [5]. Wang et al. assessed 15 patients with nonunion of the tibia treated with circular frames. After a mean of 12 months, bone union was achieved in $100 \%$ of cases [17]. They dynamized the frame before removal for assess the mechanical stability of the new bone. They removed fixator when the nonunion was corticolized on 3 of 4 sides in radiographs. After external fixator removal, they applied functional brace for at least 4 weeks [17]. Yin achieved union in all of the 60 patients with nonunion of the tibia treated with an Ilizarov external fixator [21]. Yin removed external fixator when radiographs showed a minimum of three complete cortices [21].

We believe that very good outcome in our patients (refracture only in $4.9 \%$ of patients) are related to our treatment protocol. We have minimized the risk of re-fracture through delayed frame removal and weight-bearing protocol. The average time of Ilizarov frame removal was 7.9 months. Ilizarov external fixator was removed once union of the nonunion was confirmed radiographically and clinically. The weight-bearing protocol is also important. Loading of the limb was gradually increased, depending on the degree of bone remodeling at the site of nonunion visualized with radiography.

The authors presented papers in which they evaluated from 8 to 94 patients treated with circular frames due to nonunion of the tibia [2-27, 30, 32]. In our work, we evaluated a group of 102 patients. All patients in our study achieved bone union, which constitutes a better outcome than those reported on average in the literature $(73.7-100 \%)[1-27,30$, 32]. Callus formation and bone union tend to take a longer time in patients with nonunion of the tibia $[10,13]$. This extends treatment duration (the length of time which the Ilizarov apparatus remains on the treated limb) in comparison to that in patients who undergo corrective surgeries, such as limb lengthening [10,13]. This is another reason why patients with nonunion of the tibia treated with the Ilizarov method should be followed up for a longer time and why long-term treatment outcomes should be assessed. The mean length of time which the Ilizarov external fixator was in place in our patients was 7.9 months, which is consistent with the data from the literature (as the reported treatment duration ranged from 5.8 to 13.5 months) $[3,4,7-11,14$, $17,25]$.

None of the studies mentioned above [1-27, 30-33] assessed the proportion of patients with maintained bone union. In our study, maintained bone union was observed in $95.1 \%$ of patients at the follow-up visit at least 2 years after treatment, which indicates excellent long-term treatment outcomes in nonunion of the tibia treated with the Ilizarov method.

Four out of five patients with re-fracture had infected, atrophic nonunion in $1 / 3$ distal of tibia. All of patients with re-fracture had close surgery technique. Infection, atrophic nonunion, nonunion in $1 / 3$ distal of tibia, and close surgery 
technique are a risk factors of re-fracture after tibia nonunion treatment with Ilizarov method. All of the re-fracture patients had poor results in ASAMI Bone Score and ASAMI Functional Score. Re-fracture has a negative effect on the final outcome in comparison to cases with maintained bone union.

\section{Conclusions}

Since long-term treatment outcomes are the most importance to both the patients and the surgeons, we suggest that maintained union rates be introduced as a new assessment criterion of long-term outcomes. It is both the union rates (short term, achieving union after treatment) and maintained union rates (long term) that need to be assessed as part of any comprehensive evaluation of Ilizarov treatment outcomes in patients with nonunion of the tibia.

The Ilizarov method helps to achieve very good shortterm and long-term outcomes both in the treatment of nonunion of the tibia.

Infection, atrophic nonunion, nonunion in $1 / 3$ distal of tibia, and close surgery technique are a risk factors of refracture after tibia nonunion treatment with Ilizarov method.

Funding There is no funding source.

\section{Compliance with ethical standards}

Conflict of interest The authors declare that they have no conflict of interest.

Ethical approval This article does not contain any studies with human participants or animals performed by any of the authors.

Open Access This article is licensed under a Creative Commons Attribution 4.0 International License, which permits use, sharing, adaptation, distribution and reproduction in any medium or format, as long as you give appropriate credit to the original author(s) and the source, provide a link to the Creative Commons licence, and indicate if changes were made. The images or other third party material in this article are included in the article's Creative Commons licence, unless indicated otherwise in a credit line to the material. If material is not included in the article's Creative Commons licence and your intended use is not permitted by statutory regulation or exceeds the permitted use, you will need to obtain permission directly from the copyright holder. To view a copy of this licence, visit http://creativecommons.org/licenses/by/4.0/.

\section{References}

1. Yin P, Ji Q, Li T, Li J, Li Z, Liu J, Wang G, Wang S, Zhang L, Mao Z, Tang P (2015) A systematic review and meta-analysis of Ilizarov methods in the treatment of infected nonunion of tibia and femur. PLoS ONE 10(11):e0141973. https://doi.org/10.1371/journ al.pone. 0141973

2. Peng J, Min L, Xiang Z, Huang F, Tu C, Zhang H (2015) Ilizarov bone transport combined with antibiotic cement spacer for infected tibial nonunion. Int J Clin Exp Med 8(6):10058-10065

3. Schoenleber SJ, Hutson JJ Jr (2015) Treatment of hypertrophic distal tibia nonunion and early malunion with callus distraction. Foot Ankle Int 36(4):400-407. https://doi.org/10.1177/10711 00714558509

4. Zhang H, Xue F, Jun Xiao H (2018) Ilizarov method in combination with autologous mesenchymal stem cells from iliac crest shows improved outcome in tibial non-union. Saudi J Biol Sci 25(4):819-825. https://doi.org/10.1016/j.sjbs.2016.11.001

5. Meleppuram JJ, Ibrahim S (2016) Experience in fixation of infected non-union tibia by Ilizarov technique - a retrospective study of 42 cases. Rev Bras Ortop 52(6):670-675. https://doi. org/10.1016/j.rboe.2016.11.008

6. Wani NB, Syed B (2015) Ilizarov ring fixator in the management of infected non-unions of tibia. SICOT J 1:22. https://doi. org/10.1051/sicotj/2015022

7. Abuomira IE, Sala F, Elbatrawy Y, Lovisetti G, Alati S, Capitani D (2016) Distraction osteogenesis for tibial nonunion with bone loss using combined Ilizarov and Taylor spatial frames versus a conventional circular frame. Strateg Trauma Limb Reconstr 11(3):153-159

8. Baruah RK (2007) Ilizarov methodology for infected non union of the tibia: classic circular transfixion wire assembly vs. hybrid assembly. Indian J Orthop 41:198-203. https://www.ijoonline. com/text.asp?2007/41/3/198/33682. (serial online, cited 2019 Jan 14)

9. Eralp İL, Kocaoğlu M, Dikmen G, Azam ME, Balcı Hİ, Bilen FE (2016) Treatment of infected nonunion of the juxta-articular region of the distal tibia. Acta Orthop Traumatol Turc 50(2):139 146. https://doi.org/10.3944/AOTT.2015.15.0147

10. Hosny G, Shawky MS (1998) The treatment of infected nonunion of the tibia by compression-distraction techniques using the Ilizarov external fixator. Int Orthop 22(5):298-302

11. Khan MS, Rashid H, Umer M, Qadir I, Hafeez K, Iqbal A (2015) Salvage of infected non-union of the tibia with an Ilizarov ring fixator. J Orthop Surg (Hong Kong) 23(1):52-55

12. Madhusudhan TR, Ramesh B, Manjunath K, Shah HM, Sundaresh DC, Krishnappa N (2008) Outcomes of Ilizarov ring fixation in recalcitrant infected tibial non-unions-a prospective study. J Trauma Manag Outcomes 2(1):6. https://doi. org/10.1186/1752-2897-2-6

13. Magadum MP, Basavaraj Yadav CM, Phaneesha MS, Ramesh LJ (2006) Acute compression and lengthening by the Ilizarov technique for infected nonunion of the tibia with large bone defects. J Orthop Surg (Hong Kong) 14(3):273-279

14. Sahu RL, Ranjan R (2016) Treatment of complex nonunion of the shaft of the tibia using Ilizarov technique and its functional outcome. Niger Med J 57(2):129-133. https://doi.org/10.4103/03001652.182076

15. Sanders DW, Galpin RD, Hosseini M, MacLeod MD (2002) Morbidity resulting from the treatment of tibial nonunion with the Ilizarov frame. Can J Surg 45(3):196-200

16. Shahid M, Hussain A, Bridgeman P, Bose D (2013) Clinical outcomes of the Ilizarov method after an infected tibial non union. Arch Trauma Res 2(2):71-75. https://doi.org/10.5812/atr.11300. (Epub 2013 Aug 1)

17. Wang H, Wei X, Liu P, Fu YH, Wang PF, Cong YX, Zhang BF, Li Z, Lei JL, Zhang K, Zhuang Y (2017) Quality of life and complications at the different stages of bone transport for treatment infected nonunion of the tibia. Medicine 96(45):e8569. https://doi. org/10.1097/MD.0000000000008569 
18. Yin P, Zhang L, Li T, Zhang L, Wang G, Li J, Liu J, Zhou J, Zhang Q, Tang P (2015) Infected nonunion of tibia and femur treated by bone transport. J Orthop Surg Res 10:49. https://doi.org/10.1186/ s13018-015-0189-5

19. McNally M, Ferguson J, Kugan R, Stubbs D (2017) Ilizarov treatment protocols in the management of infected nonunion of the tibia. J Orthop Trauma 31(Suppl 5):S47-S54

20. Dróżdż M, Rak S, Bartosz P, Białecki J, Marczyński W (2017) Results of the treatment of infected nonunions of the lower limbs using the Ilizarov method. Ortop Traumatol Rehabil 19(2):111-125

21. Yin P, Zhang Q, Mao Z, Li T, Zhang L, Tang P (2014) The treatment of infected tibial nonunion by bone transport using the Ilizarov external fixator and a systematic review of infected tibial nonunion treated by Ilizarov methods. Acta Orthop Belg 80(3):426-435

22. Xu J, Jia YC, Kang QL, Chai YM (2015) Management of hypertrophic nonunion with failure of internal fixation by distraction osteogenesis. Injury 46(10):2030-2035. https://doi.org/10.1016/j. injury.2015.06.020. (Epub 2015 Jun 17)

23. Marsh DR, Shah S, Elliott J, Kurdy N (1997) The Ilizarov method in nonunion, malunion and infection of fractures. J Bone Jt Surg $\operatorname{Br}$ 79(2):273-279

24. Megas P, Saridis A, Kouzelis A, Kallivokas A, Mylonas S, Tyllianakis M (2010) The treatment of infected nonunion of the tibia following intramedullary nailing by the Ilizarov method. Injury 41(3):294-299. https://doi.org/10.1016/j.injury.2009.09.013

25. Laursen MB, Lass P, Christensen KS (2000) Ilizarov treatment of tibial nonunions results in 16 cases. Acta Orthop Belg 66(3):279-285

26. Hosny GA, Ahmed AA, Hussein MA (2018) Clinical outcomes with the corticotomy-first technique associated with the Ilizarov method for the management of the septic long bones non-union. Int Orthop 42(12):2933-2939. https://doi.org/10.1007/s0026 4-018-3924-9. (Epub 2018 Apr 7)
27. Morandi M, Zembo MM, Ciotti M (1989) Infected tibial pseudarthrosis. A 2-year follow up on patients treated by the Ilizarov technique. Orthopedics 12(4):497-508

28. Paley D, Catagni MA, Argnani F, Villa A, Benedetti GB, Cattaneo R (1989) Ilizarov treatment of tibial nonunions with bone loss. Clin Orthop Relat Res 241:146-165

29. Catagni MA (1991) Classification and treatment of nonunion. In: Maiocchi AB, Aronson J (eds) Operative principles of Ilizarov by ASAMI group. Williams and Wilkins, Baltimore, pp 190-198

30. Cunningham BA, O'Connor DP, Brinker MR (2018) High rate of union for aseptic distal tibial nonunions in adults treated with slow gradual compression via circular external fixation. J Orthop Trauma 32(6):301-305. https://doi.org/10.1097/BOT.0000000000 001141

31. Napora JK, Weinberg DS, Eagle BA, Kaufman BR, Sontich JK (2018) Hexapod stacked transport for tibial infected nonunions with bone loss: long-term functional outcomes. J Orthop Trauma 32(1):e12-e18. https://doi.org/10.1097/BOT.0000000000001005

32. Bhardwaj R, Singh J, Kapila R, Boparai RS (2019) Comparison of Ilizarov ring fixator and rail fixator in infected nonunion of long bones: a retrospective followup study. Indian J Orthop 53(1):8288. https://doi.org/10.4103/ortho.IJOrtho_77_17

33. Jubbal KT, Zavlin D, Doval AF, Cherney SM, Brinker MR, Dinh TA, Echo A (2019) Immediate soft-tissue reconstruction for chronic infected tibia nonunions treated with an Ilizarov frame. Plast Reconstr Surg Glob Open 7(3):e2180. https://doi. org/10.1097/GOX.0000000000002180

Publisher's Note Springer Nature remains neutral with regard to jurisdictional claims in published maps and institutional affiliations. 